\title{
Population Density, Fertility, and Childcare Services From the Perspective of a Two-Region Overlapping Generations Model $^{1}$
}

\author{
Ryo Ishida \\ Visiting Scholar \\ Policy Research Institute, Ministry of Finance \\ Kazumasa Oguro \\ Professor \\ Faculty of Economics, Hosei University \\ Masaya Yasuoka \\ Associate Professor \\ School of Economics, Kwansei Gakuin University
}

\begin{abstract}
In countries confronting the issue of low fertility, as Japan is, dual trends showing higher regional population density associated with lower fertility rates are being confirmed. It is therefore an important theme for analysis to deepen discussions related to reducing regional fertility disparities by increasing fertility through the implementation of comprehensive childcare support policies, which might facilitate the striking of a balance between child-rearing and work, even in highly populated regions.

As described herein, we constructed a simple theoretical two-region Overlapping Generations (OLG) by incorporating migration and land prices. Using it, we analyzed effects of population density and childcare services on fertility. Results elucidated the following three points.

First, in the presence of congestion costs associated with increased population density, the fertility rate of the region decreases with increased population density. However, if the time cost of child-rearing is brought down by raising the level of the childcare services provided in the region, then the effect of increased population density on fertility can be restrained.

Second, when the effect of population size on productivity is less than a certain level, improvement in the childcare services raises the relative ratio of the population density. When the effect of population size on productivity exceeds a certain level, however, the relative ratio of the population density decreases if the relative ratio of the time cost of child-rearing decreases as a result of childcare service reform.

Third, where each region imposes payroll tax on its residents and uses its tax revenue as the financial resources to adopt a decentralized strategy of providing childcare services to its region, the level of childcare services that maximizes the utility of a representative agent in each region is independent of the childcare services of any other region. Therefore, manipulation of the level of childcare services becomes a dominant strategy.
\end{abstract}

JEL: H40; J13; J61; R10; R12; R23

Keywords: population density, fertility, congestion, migration, childcare services, overlapping generation (OLG)

\footnotetext{
1 We extend our appreciation to Professor XX of XX University for offering us suggestions at an early stage of the drafting of this paper. The authors take full responsibility for the wording presented herein. The entire contents herein reflect their personal opinions and do not represent the official position of any institution with which they are affiliated. Furthermore, this work was supported by JSPS KAKENHI (Grant-in-Aid for Scientific Research) Grant Numbers 15K03465 and 26380253.
} 


\section{Introduction}

The major purpose of this paper is construction of a simple theoretical two-region Overlapping Generation (OLG) model by incorporating migration and land prices and analysis of the effects of population density and childcare services on fertility.

As described by Galor and Weil (1996), economic growth increased the wage rates for female workers. The opportunity cost of child-rearing increased because women had to abandon their jobs to care for children. Consequently, child populations have continued to decline in OECD countries. Nevertheless, female workers can choose to have children without giving up their jobs if childcare services are in place. In other words, women must strike a balance between child-rearing and work. Apps and Rees (2004) demonstrated that fertility increased concomitantly with increased wage rates for female workers. The positive correlation between the female labor force participation rate and fertility in OECD countries was confirmed by Sleebos (2003). Apparently, an increased supply of female labor has the effect of making more money available for child-rearing through increased household income, thereby increasing the number of children.

Several studies have been conducted to elucidate the mutual correlation of fertility and childcare support policies. Van Groezen, Leers and Meijdam (2003) reported that a child allowance increased fertility. Aside from a child allowance, an educational assistance policy might also support families with small children. However, although an educational assistance policy is effective for decreasing child-rearing costs substantially, it also decreases fertility, as reported by Zhang (1997).

One might argue that the female labor force participation rate in OECD countries and the scale of childcare support policies contribute to disparities in fertility. In Japan, however, the fertility rate differs among prefectures. It is lower in highly populated areas where there is a preponderance of nuclear family households such as in Tokyo, although it is higher in less-populated areas where the number of nuclear family households is small. Presumably, the presence of such a gap in fertility among prefectures is affected by the availability and quality of childcare services that are provided. It is possible that although the female labor force participation rate is generally high in highly populated areas, fertility remains low because childcare centers are not fully developed, and grandparents who might be able to take care of grandchildren do not live under one roof with the child-rearing couple.

Then, would it be possible to increase the fertility rate and narrow regional fertility disparities through sufficient childcare support policies that would ensure both 
child-rearing and work even in highly populated areas? In other words, can it be said that childcare support policies that differ among regions have caused regional fertility disparities? The purpose of this paper is consideration of this issue.

Examples of analyses of regional fertility disparities include those performed by Zhang (2002), Sato and Yamamoto (2005), and Sato (2007). Whereas population integration engenders positive externality through improved labor productivity, resulting in higher wage rates, the fertility rate decreases because of increased opportunity costs of child-rearing. Given the introduction of childcare support policies, it is conceivable that higher fertility rates can be achieved in highly populated areas under the higher wage rate.

Kulu (2013) clarified through empirical study, using Finnish data, the presence of regional fertility disparities under the assumption that fertility disparities exist between big cities and small cities. Other examples of empirical studies related to fertility disparities include those performed by de Beers J., Deerenberg I. (2007), O'Connell M. (1981), and Tumen S. (2012).

This paper is organized as follows. Section 2 presents a two-region OLG model by incorporating migration and land prices to analyze the effects of population density and childcare services on fertility. Section 3 presents analyses of population density and steady state of the two regions. Section 4 describes optimal childcare services level in a case where the two regions adopt a decentralized strategy to provide childcare services entirely limited to their own regions. Finally, Section 5 presents a summary of results and describes future issues.

\section{Theoretical model}

Herein, we construct a two-region OLG model, combining migration and land prices to analyze the effects of population density and childcare services on fertility.

\section{Household}

We assume a model that consists of $j=1,2$ regions. The lifetime utility of generation $t$ residing in $j$ region is given by the following equation.

$$
U_{j}(t)=n_{j}(t+1)^{\alpha} c_{j}(t)^{\beta} z_{j}(t+1)^{\gamma} q_{j}(t)^{1-\alpha-\beta-\gamma}
$$

In that equation, $n_{j}(t+1)$ stands for the number of live births of generation $t, c_{j}(t)$ represents the consumption, $z_{j}(t+1)$ denotes the educational investment level per child, $q_{j}(t)$ stands for space consumption, and $\alpha>\gamma$ and $1-\alpha-\beta-\gamma>0$. Generation $t$ residing in region $j$ maximizes the lifetime utility given by equation (1) under the 
lifetime budget constraint as given below.

$$
z_{j}(t+1) n_{j}(t+1)+c_{j}(t)+R_{j}(t) q_{j}(t)=\left(1-\tau_{j}\right) e_{j}(t)\left(1-\xi\left(g_{j}\right) n_{j}(t+1)\right)-\Phi_{j}(t)
$$

In that equation, $R_{j}(t)$ signifies the land rent per unit of space in region $j, \xi\left(g_{j}\right)$ denotes the time cost of child-rearing per child, $g_{j}$ represents the level of childcare services, $e_{j}(t)$ stands for the wage rate, $\tau_{j}$ represents the payroll tax, and $\Phi_{j}(t)$ is the congestion cost associated with an increase in population density. In this case, the time cost of child-rearing $\xi\left(g_{j}\right)$ is assumed to decrease as childcare services $g_{j}$ increase. Briefly, it is characterized by $\partial \xi\left(g_{j}\right) / \partial g_{j}<0$ and is assumed to satisfy $\xi\left(g_{j}\right) \geq 0 \forall g_{j}$. Congestion costs are given as $\Phi_{j}(t) \equiv\left(1-\tau_{j}\right) e_{j}(t) \Omega_{j}\left(n_{j}(t+1) N_{j}(t) / L_{j}\right)$, where the regional population size in $j$ region is $n_{j}(t+1) N_{j}(t)$, the land supply is $L_{j}$, and the constant is $\Omega_{j} \geq 0$, and the upper limit of the payroll tax $\tau_{j}$ is $\bar{\tau}$. In this case, each household acts to maximize utility by considering that the congestion cost $\Phi_{j}(t)$ is a given constant.

Given the premises presented above, the conditions for optimizing the lifetime utility of generation $t$ residing in region $j$ are given by the following equations.

$$
\begin{aligned}
& n_{j}(t+1)=\frac{(\alpha-\gamma)}{(1-\gamma) \xi\left(g_{j}\right)+(\alpha-\gamma) \Omega_{j} N_{j}(t) / L_{j}} \\
& z_{j}(t+1)=\frac{\gamma}{\alpha-\gamma}\left(1-\tau_{j}\right) e_{j}(t) \xi\left(g_{j}\right) \\
& c_{j}(t)=\frac{\beta\left(1-\tau_{j}\right) e_{j}(t) \xi\left(g_{j}\right)}{(1-\gamma) \xi\left(g_{j}\right)+(\alpha-\gamma) \Omega_{j} N_{j}(t) / L_{j}} \\
& \left.q_{j}(t)=\frac{(1-\alpha-\beta-\gamma)\left(1-\tau_{j}\right) e_{j}(t) \xi\left(g_{j}\right)}{R_{j}(t)\left[(1-\gamma) \xi\left(g_{j}\right)+(\alpha-\gamma) \Omega_{j} N_{j}(t) / L_{j}\right.}\right]
\end{aligned}
$$

\section{Production}

Next, the production function in region $j$ is assumed to consist of productivity $e_{j}(t)$ and labor force $\Xi_{j}(t)$, which are influenced by population size excluding children $N_{j}(t)$ to reflect the effect of population integration, and is therefore given as shown below.

$$
Y_{j}(t)=e_{j}(t) \Xi_{j}(t)
$$

\section{Childcare services and decentralized strategy of each region}

As described in this paper, the level of childcare services $g_{j}$ represents the number of childcare staff allocated by daycare centers per household: it is assumed to satisfy $0 \leq g_{j} \leq 1$ and $\xi(1) \geq 0$. Furthermore, we assume that a decentralized strategy is taken, where each $j$ region imposes payroll tax $\tau_{j}$ on its residents and uses its tax revenue as the financial resources to provide childcare services $g_{j}$ to its region. In this 
case, each $j$ region employs childcare staff from among the regional residents at wage rate $e_{j}(t)$. Then, the labor supplied by childcare staff is equated with the labor supplied by workers in the productive sector. Therefore $l_{j}(t) \equiv\left(1-\xi\left(g_{j}\right) n_{j}(t+1)\right)$. The budget constraint of the public sector of each $j$ region is given by the following equation $(j=1,2)$ as

$$
g_{j} e_{j}(t) l_{j}(t) N_{j}(t)=\tau_{j} e_{j}(t) l_{j}(t) N_{j}(t) .
$$

If it is assumed that the childcare staff do not contribute to the productive sector, then the labor market equilibrium of each $j$ region is defined as follows $(j=1,2)$.

$$
\Xi_{j}(t)=l_{j}(t) N_{j}(t)\left(1-g_{j}\right)
$$

\section{Land market equilibrium}

From equation (6), the land demand in region $j$ is $q_{j}(t) N_{j}(t)$. If the land supply is $L_{j}$, then according to the equilibrium conditions related to demand and supply of land defined by $q_{j}(t) N_{j}(t)=L_{j}$, the land rent per unit of space in $j$ region is determined as shown below.

$$
R_{j}(t)=\frac{1-\alpha-\beta-\gamma}{(1-\gamma) \xi\left(g_{j}\right)+(\alpha-\gamma) \Omega_{j} N_{j}(t) / L_{j}}\left(N_{j}(t) / L_{j}\right)\left(1-\tau_{j}\right) e_{j}(t) \xi\left(g_{j}\right)
$$

\section{Migration equilibrium}

From equation (3), substituting equation (6) into the utility function of equation (1), the indirect utility function of generation $t$ residing in $j$ region is obtainable as

$$
U_{j}(t) \propto \bar{U}_{j}(t)=\frac{\left(\left(1-\tau_{j}\right) e_{j}(t) \xi\left(g_{j}\right)\right)^{1-\alpha}}{\left((1-\gamma) \xi\left(g_{j}\right)+(\alpha-\gamma) \Omega_{j} N_{j}(t) / L_{j}\right)^{1-\gamma} R_{j}(t)^{1-\alpha-\beta-\gamma}} .
$$

If the indirect utility function of $j=1$ region is higher than that of the $j=2$ region, then migration from $j=2$ region to $j=1$ region will occur. If the indirect utility function of $j=2$ region is higher than that of the $j=1$ region, then migration from $j=1$ region to $j=2$ region will occur. Therefore, if the total population of generation $t$ is $N^{\text {total }}(t)$, then the following equation becomes valid in terms of the migration equilibrium.

$$
\begin{aligned}
& N_{1}(t)+N_{2}(t)=N^{\text {total }}(t) \\
& \bar{U}_{1}(t)=\bar{U}_{2}(t)
\end{aligned}
$$

Now, substituting equation (10) into equation (11), equation (13) becomes the following. 


$$
\frac{\left(\left(1-\tau_{1}\right) e_{1}(t) \xi\left(g_{1}\right)\right)^{\beta+\gamma}}{\left(\xi\left(g_{1}\right)+\frac{\alpha-\gamma}{1-\gamma} \Omega_{1} N_{1}(t) / L_{1}\right)^{\alpha+\beta}\left(N_{1}(t) / L_{1}\right)^{1-\alpha-\beta-\gamma}}=\frac{\left(\left(1-\tau_{2}\right) e_{2}(t) \xi\left(g_{2}\right)\right)^{\beta+\gamma}}{\left(\xi\left(g_{2}\right)+\frac{\alpha-\gamma}{1-\gamma} \Omega_{2} N_{2}(t) / L_{2}\right)^{\alpha+\beta}\left(N_{2}(t) / L_{2}\right)^{1-\alpha-\beta-\gamma}}
$$

Then the population of each region obtained from simultaneous equations (12) and (14) is defined as shown below.

$$
\begin{aligned}
& N_{1}(t)=\frac{1}{1+\theta\left(t, g_{1}, g_{2}\right)} N^{\text {total }}(t) \\
& N_{2}(t)=\frac{\theta\left(t, g_{1}, g_{2}\right)}{1+\theta\left(t, g_{1}, g_{2}\right)} N^{\text {total }}(t)
\end{aligned}
$$

From equations (3) and (15), the generation population dynamics is given as follows.

$$
\begin{aligned}
N^{\text {total }}(t+1)= & n_{1}(t+1) N_{1}(t)+n_{2}(t+1) N_{2}(t) \\
= & \frac{\alpha-\gamma}{1-\gamma}\left[\frac{1}{\left(\xi\left(g_{1}\right)+\frac{\alpha-\gamma}{1-\gamma} \Omega_{1} N_{1}(t) / L_{1}\right)}+\frac{\theta\left(t, g_{1}, g_{2}\right)}{\left(\xi\left(g_{2}\right)+\frac{\alpha-\gamma}{1-\gamma} \Omega_{2} N_{2}(t) / L_{2}\right)}\right] \frac{N^{\text {total }}(t)}{1+\theta\left(t, g_{1}, g_{2}\right)}(16)
\end{aligned}
$$

\section{Analysis}

This section presents analyses of the models described in the preceding section.

In a simple case in which productivity $e_{j}(t)$ of equation (7) is independent of population size $N_{j}(t)$, equation (14) can be transformed as follows.

$$
\left(\frac{\xi\left(g_{2}\right)+\frac{\alpha-\gamma}{1-\gamma} \Omega_{2} N_{2}(t) / L_{2}}{\xi\left(g_{1}\right)+\frac{\alpha-\gamma}{1-\gamma} \Omega_{1} N_{1}(t) / L_{1}}\right)^{\alpha+\beta}\left(\frac{N_{2}(t) / L_{2}}{N_{1}(t) / L_{1}}\right)^{1-\alpha-\beta-\gamma}=\left(\frac{\left(1-\tau_{2}\right) e_{2}(t) \xi\left(g_{2}\right)}{\left(1-\tau_{1}\right) e_{1}(t) \xi\left(g_{1}\right)}\right)^{\beta+\gamma}
$$

This equation represents the relation between the population density of $j=1$ region and that of $j=2$ region. It shows that it is affected by the relative ratio of the post-tax wage rate and time cost of child-rearing under a certain childcare services level. Therefore, we can obtain the following proposition.

Proposition 1. The relation of population density between the two regions is determined by equation (17) when productivity $e_{j}(t)$ is independent of population size $N_{j}(t)$. The relative ratio of the population density of both regions is given as follows, especially when $\Omega_{j}=0(j=1,2)$.

$$
\frac{N_{2}(t) / L_{2}}{N_{1}(t) / L_{1}}=\left[\left(\frac{\left(\left(1-\tau_{2}\right) e_{2}(t)\right.}{\left(1-\tau_{1}\right) e_{1}(t)}\right)^{\beta+\gamma}\left(\frac{\xi\left(g_{1}\right)}{\xi\left(g_{2}\right)}\right)^{\alpha-\gamma}\right]^{\frac{1}{1-\alpha-\beta-\gamma}}
$$


Equation (17) includes various information. For instance, let us fix variables other than $N_{j}(t)(j=1,2)$ and increase only $\tau_{1}$. In this case, from equations (12) and (17), $N_{1}(t)$ must decrease and $N_{2}(t)$ must increase. In other words, when $i \neq j$, $\partial\left(N^{N} / L_{i}\right) / \partial \tau_{i}<0$ and $\partial\left(N / L_{L}\right) / \partial \tau_{j}>0$ are valid, which means that increased payroll tax in a region will decrease the population density in the corresponding local region, but that the increased payroll tax in another region will increase the population density in its own local region. Similarly, $\partial\left({ }^{N} / L_{1}\right) / \partial e_{i}>0$ and $\partial\left({ }^{N} / L_{i}\right) / \partial e_{j}<0$ are valid, which means that increased productivity in a region will increase the population density in the corresponding local region, but that the increased productivity in another region will decrease the population density of its own local region.

As for the congestion cost coefficient, " $\partial\left({ }^{N} / L_{L}\right) / \partial \Omega_{i}<0$ and $\partial\left({ }^{N} / L_{L}\right) / \partial \Omega_{j}>0$ " are valid, which means that the increased congestion cost in a region will decrease the population density in the corresponding local region, but that the increased congestion cost in another region will increase population density in its own local region.

Furthermore, equation (17) can be transformed as presented below.

$$
\left(\frac{1+\frac{\alpha-\gamma}{1-\gamma}\left(\Omega_{2} N_{2}(t) / L_{2}\right) / \xi\left(g_{2}\right)}{1+\frac{\alpha-\gamma}{1-\gamma}\left(\Omega_{1} N_{1}(t) / L_{1}\right) / \xi\left(g_{1}\right)}\right)^{\alpha+\beta}\left(\frac{\xi\left(g_{2}\right)}{\xi\left(g_{1}\right)}\right)^{\alpha-\gamma}\left(\frac{N_{2}(t) / L_{2}}{N_{1}(t) / L_{1}}\right)^{1-\alpha-\beta-\gamma}=\left(\frac{\left(1-\tau_{2}\right) e_{2}(t)}{\left(1-\tau_{1}\right) e_{1}(t)}\right)^{\beta+\gamma}
$$

When $\xi\left(g_{j}\right)>>\Omega_{j} N_{j}(t) / L_{j}(j=1,2)$ is valid, “ $\partial\left({ }^{N} / L_{1}\right) / \partial \xi_{i}<0$ and $\partial\left(N / L_{L}\right) / \partial \xi_{j}>0 "$ are valid for the time cost of child-rearing, which means that the increased time cost of child-rearing in a region will decrease population density in the corresponding local region, but that the increased time cost of child-rearing in another region will increase population density in its own local region. It is noteworthy that the population density might increase if an increase in the post-tax wage rate occurs because increased productivity more than offsets the increased time cost of child-rearing.

However, when $\xi\left(g_{j}\right)<<\Omega_{j} N_{j}(t) / L_{j}(j=1,2)$, similarly “ $\partial\left({ }^{N} / L_{i}\right) / \partial \xi_{i}>0$ and $\partial\left(N^{N} / L_{i}\right) / \partial \xi_{j}<0 "$ are valid for the time cost of child-rearing, which will bring about the following phenomena: increased time cost of child-rearing in a region will increase the population density of the corresponding local region but increased time cost of child-rearing in another region will decrease the population density of its own local region. This result might appear peculiar, but it is true because of a fallacy of composition because each household takes congestion cost $\Phi_{j}(t)$ as given. ${ }^{2}$

\footnotetext{
2 When the time cost of child-rearing $\xi$ decreases, the utility is expected to increase always if other conditions are fixed. In reality, however, as the fertility rate increases and the congestion cost increases (since individuals engage in optimization behavior by considering that the congestion cost is constant), the utility can instead decrease.
} 
Next, we examine a case in which productivity $e_{j}(t)$ is dependent on population $N_{j}(t)$, and where productivity is definable by $e_{j}(t)=a_{j}\left(N_{j}(t) / L_{j}\right)^{\rho}$. In this instance, equation (14) can be transformed as shown below.

$$
\frac{\left\{\left(1-\tau_{1}\right) a_{1}\left(N_{1}(t) / L_{1}\right)^{\rho} \xi\left(g_{1}\right)\right\}^{\beta+\gamma}}{\left(\xi\left(g_{1}\right)+\frac{\alpha-\gamma}{1-\gamma} \Omega_{1} N_{1}(t) / L_{1}\right)^{\alpha+\beta}\left(N_{1}(t) / L_{1}\right)^{1-\alpha-\beta-\gamma}}=\frac{\left\{\left(1-\tau_{2}\right) a_{2}\left(N_{2}(t) / L_{2}\right)^{\rho} \xi\left(g_{2}\right)\right\}^{\beta+\gamma}}{\left(\xi\left(g_{2}\right)+\frac{\alpha-\gamma}{1-\gamma} \Omega_{2} N_{2}(t) / L_{2}\right)^{\alpha+\beta}\left(N_{2}(t) / L_{2}\right)^{1-\alpha-\beta-\gamma}}
$$

From this equation, the relative ratio of the population density is given as follows.

$$
\left(\frac{\xi\left(g_{2}\right)+\frac{\alpha-\gamma}{1-\gamma} \Omega_{2} N_{2}(t) / L_{2}}{\xi\left(g_{1}\right)+\frac{\alpha-\gamma}{1-\gamma} \Omega_{1} N_{1}(t) / L_{1}}\right)^{\alpha+\beta}\left(\frac{N_{2}(t) / L_{2}}{N_{1}(t) / L_{1}}\right)^{1-\alpha-(1+\rho)(\beta+\gamma)}=\left(\frac{\left(1-\tau_{2}\right) a_{2} \xi\left(g_{2}\right)}{\left(1-\tau_{1}\right) a_{1} \xi\left(g_{1}\right)}\right)^{\beta+\gamma}
$$

Proposition 2. When productivity $e_{j}(t)$ is dependent on population size $N_{j}(t)$, and productivity is $e_{j}(t)=a_{j}\left(N_{j}(t) / L_{j}\right)^{\rho}$, the relation of population density of both regions is defined by equation (18). The relative ratio of the population density of both regions is given as follows, especially when $\Omega_{j}=0(j=1,2)$.

$$
\frac{N_{2}(t) / L_{2}}{N_{1}(t) / L_{1}}=\left[\left(\frac{\xi\left(g_{1}\right)}{\xi\left(g_{2}\right)}\right)^{\alpha-\gamma}\left(\frac{\left(1-\tau_{2}\right) a_{2}}{\left(1-\tau_{1}\right) a_{1}}\right)^{\beta+\gamma}\right]^{\frac{1}{1-\alpha-(1+\rho)(\beta+\gamma)}}
$$

In equation (19), $\Gamma_{1}(\rho) \equiv 1-\alpha-(1+\rho)(\beta+\gamma)$, where the direction of the relative ratio of the population density is determined by sign $\Gamma_{1}(\rho)$ when $\Omega_{j}=0 \quad(j=1,2)$, the relation between the relative ratio of the population density and time cost of child-rearing and between the relative ratio of the population density and payroll tax can be reversed by sign $\Gamma_{1}(\rho)$. For instance, when the effect of population size on productivity is less than a certain amount and satisfies $\rho<(1-\alpha) /(\beta+\gamma)-1$, the relative ratio of the population density will increase if the relative ratio of the time cost of child-rearing decreases because of childcare service reform (alternatively if the relative ratio of $\left(1-\tau_{j}\right)$ increases attributable to tax system reform). Conversely, if the effect of population size on productivity exceeds a certain level and satisfies $\rho>(1-\alpha) /(\beta+\gamma)-1$, then the relative ratio of population density will increase even if the relative ratio of the time cost of child-rearing increases because of a delay in childcare service reform (alternatively if

When $\Omega$ is small, such a phenomenon is less likely to occur (a fallacy of composition can never occur when $\Omega=0$ ). When $\Omega$ is large, such phenomena are more likely to occur. 
the relative ratio of $\left(1-\tau_{j}\right)$ decreases because of tax system reform) because the effect of population size on productivity is overwhelmingly strong. Furthermore, if the relative ratio of the time cost of child-rearing decreases attributable to childcare service reform, then the relative ratio of the population density is expected to decrease.

Because equation (18) can be transformed as follows, the above argument is valid even when $\xi\left(g_{j}\right)>>\Omega_{j} N_{j}(t) / L_{j}$.

$$
\left(\frac{1+\frac{\alpha-\gamma}{1-\gamma}\left(\Omega_{2} N_{2}(t) / L_{2}\right) / \xi\left(g_{2}\right)}{1+\frac{\alpha-\gamma}{1-\gamma}\left(\Omega_{1} N_{1}(t) / L_{1}\right) / \xi\left(g_{1}\right)}\right)^{\alpha+\beta}\left(\frac{\xi\left(g_{2}\right)}{\xi\left(g_{1}\right)}\right)^{\alpha-\gamma}\left(\frac{N_{2}(t) / L_{2}}{N_{1}(t) / L_{1}}\right)^{1-\alpha-(1+\rho)(\beta+\gamma)}=\left(\frac{\left(1-\tau_{2}\right) a_{2}}{\left(1-\tau_{1}\right) a_{1}}\right)^{\beta+\gamma}
$$

Equation (18) can also be transformed as shown below.

$$
\left(\frac{\frac{\alpha-\gamma}{1-\gamma}+\xi\left(g_{2}\right) /\left(\Omega_{2} N_{2}(t) / L_{2}\right)}{\frac{\alpha-\gamma}{1-\gamma}+\xi\left(g_{1}\right) /\left(\Omega_{1} N_{1}(t) / L_{1}\right)}\right)^{\alpha+\beta}\left(\frac{\Omega_{2}}{\Omega_{1}}\right)^{\alpha+\beta}\left(\frac{N_{2}(t) / L_{2}}{N_{1}(t) / L_{1}}\right)^{1+\beta-(1+\rho)(\beta+\gamma)}=\left(\frac{\left(1-\tau_{2}\right) a_{2} \xi\left(g_{2}\right)}{\left(1-\tau_{1}\right) a_{1} \xi\left(g_{1}\right)}\right)^{\beta+\gamma}
$$

As this equation suggests, when $\xi\left(g_{j}\right)<<\Omega_{j} N_{j}(t) / L_{j}$, the direction of the relative ratio of population density is determined by sign $\Gamma_{2}(\rho) \equiv 1+\beta-(1+\rho)(\beta+\gamma)$. Therefore, the summary shown in the table below is valid, similar to the discussions in the case where

\begin{tabular}{|c|c|c|c|}
\hline & $\Gamma_{1}(\rho)>0$ & $\Gamma_{1}(\rho)<0 \quad$ and $\Gamma_{2}(\rho)>0$ & $\Gamma_{2}(\rho)<0$ \\
\hline$\xi\left(g_{j}\right)>>\Omega_{j} N_{j}(t) / L_{j}$ & $\begin{array}{l}\text { Tax } \uparrow \Rightarrow \text { Population density (-) } \\
\text { Productivity } \uparrow \Rightarrow \text { Population } \\
\text { density (+) } \\
\text { Child-rearing cost } \uparrow \Rightarrow \text { Population } \\
\text { density (-) }\end{array}$ & $\begin{array}{l}\text { Tax } \uparrow \Rightarrow \text { Population density (+) } \\
\text { Productivity } \uparrow \Rightarrow \text { Population density (-) } \\
\text { Child-rearing cost } \uparrow \Rightarrow \text { Population } \\
\text { density }(+)\end{array}$ & $\begin{array}{l}\text { Tax } \uparrow \Rightarrow \text { Population density (+) } \\
\text { Productivity } \uparrow \Rightarrow \text { Population density } \\
(-) \\
\text { Child-rearing cost } \uparrow \Rightarrow \text { Population } \\
\text { density (+) }\end{array}$ \\
\hline$\xi\left(g_{j}\right)<<\Omega_{j} N_{j}(t) / L_{j}$ & $\begin{array}{l}\text { Tax } \uparrow \Rightarrow \text { Population density (-) } \\
\text { Productivity } \uparrow \Rightarrow \text { Population } \\
\text { density (+) } \\
\text { Child-rearing cost } \uparrow \Rightarrow \text { Population } \\
\text { density (+) }\end{array}$ & $\begin{array}{l}\text { Tax } \uparrow \Rightarrow \text { Population density (-) } \\
\text { Productivity } \uparrow \Rightarrow \text { Population density } \\
(+) \\
\text { Child-rearing cost } \uparrow \Rightarrow \text { Population } \\
\text { density (+) }\end{array}$ & $\begin{array}{l}\text { Tax } \uparrow \Rightarrow \text { Population density (+) } \\
\text { Productivity } \uparrow \Rightarrow \text { Population density } \\
(-) \\
\text { Child-rearing cost } \uparrow \Rightarrow \text { Population } \\
\text { density (-) }\end{array}$ \\
\hline
\end{tabular}
productivity $e_{j}(t)$ is independent of the population size $N_{j}(t)$.

Next we consider a "steady state" in which the gross population growth rate is $\bar{n}$ in equation (16) under a steady state. Then, $t$, after a sufficient lapse of time, is $N^{\text {total }}(t)=N^{\text {total }}\left(t_{0}\right) \bar{n}^{\left(t-t_{0}\right)}$. Furthermore, because $N_{1}(t)$ and $N_{2}(t)$ are symmetrical, the following equations become valid from equations (12), (16), and (18) if limitation " $0 \leq \theta \leq G$ " is imposed on the limit $\theta$ of $\theta(t)$, by assuming $G$ as a sufficiently large value. 


$$
\left\{\begin{array}{l}
\bar{n}=\frac{\alpha-\gamma}{1-\gamma}\left[\frac{1}{\left(\xi\left(g_{1}\right)(1+\theta)+\frac{\alpha-\gamma}{1-\gamma} \Omega_{1} N^{\text {total }}\left(t_{0}\right) \bar{n}^{\left(t-t_{0}\right)} / L_{1}\right)}+\frac{\theta}{\left(\xi\left(g_{2}\right)(1+\theta)+\frac{\alpha-\gamma}{1-\gamma} \Omega_{2} N^{\text {total }}\left(t_{0}\right) \bar{n}^{\left(t-t_{0}\right)} \theta / L_{2}\right)}\right] \\
\left(\frac{\xi\left(g_{2}\right)(1+\theta)+\frac{\alpha-\gamma}{1-\gamma} \Omega_{2} N^{\text {total }}\left(t_{0}\right) \bar{n}^{\left(t-t_{0}\right)} \theta / L_{2}}{\xi\left(g_{1}\right)(1+\theta)+\frac{\alpha-\gamma}{1-\gamma} \Omega_{1} N^{\text {total }}\left(t_{0}\right) \bar{n}^{\left(t-t_{0}\right)} / L_{1}}\right)^{\alpha+\beta}\left(\frac{\theta / L_{2}}{1 / L_{1}}\right)^{1-\alpha-(1+\rho)(\beta+\gamma)}=\left(\frac{\left(1-\tau_{2}\right) a_{2} \xi\left(g_{2}\right)}{\left(1-\tau_{1}\right) a_{1} \xi\left(g_{1}\right)}\right)^{\beta+\gamma}
\end{array}\right.
$$

Three conceivable cases exist for $\bar{n}$ in equations (20) and (21): $\bar{n}>1, \bar{n}=1$, and $\bar{n}<1$. However, when $\Omega_{j}>0$, the limit of the right-hand side of equation (20) is zero in the case of $\bar{n}>1$, the left-hand side of equation (20) is $\bar{n}$ and conflicting. Therefore, when $\Omega_{j}>0$, a steady state does not exist in the case of $\bar{n}>1$. However, for $\bar{n}<1$, from the limit in equations (20) and (21), $\bar{n}$ is given as (the same also applies to the case in which $\Omega_{j}=0$ and $\bar{n} \geq 1$ )

$$
\bar{n}=\frac{\alpha-\gamma}{1-\gamma}\left[\frac{1}{\xi\left(g_{1}\right)(1+\theta)}+\frac{\theta}{\xi\left(g_{2}\right)(1+\theta)}\right],
$$

where $\theta=\frac{L_{2}}{L_{1}}\left[\left(\frac{\left(1-\tau_{2}\right) a_{2}}{\left(1-\tau_{1}\right) a_{1}}\right)^{\beta+\gamma}\left(\frac{\xi\left(g_{1}\right)}{\xi\left(g_{2}\right)}\right)^{\alpha-\gamma}\right]^{\frac{1}{1-\alpha-(1+\rho)(\beta+\gamma)}}$.

Furthermore, in the case of $\bar{n}=1$, if the population of each region in a steady state is $\bar{N}_{j}(j=1,2)$, then the following equation becomes valid, similar to equations (20) and (21).

$$
\left\{\begin{array}{l}
\bar{N}_{1}+\bar{N}_{2}=\frac{\alpha-\gamma}{1-\gamma}\left[\frac{\bar{N}_{1}}{\left(\xi\left(g_{1}\right)+\frac{\alpha-\gamma}{1-\gamma} \Omega_{1} \bar{N}_{1} / L_{1}\right)}+\frac{\bar{N}_{2}}{\left(\xi\left(g_{2}\right)+\frac{\alpha-\gamma}{1-\gamma} \Omega_{2} \bar{N}_{2} / L_{2}\right)}\right] \\
\left(\frac{\xi\left(g_{2}\right)+\frac{\alpha-\gamma}{1-\gamma} \Omega_{2} \bar{N}_{2} / L_{2}}{\xi\left(g_{1}\right)+\frac{\alpha-\gamma}{1-\gamma} \Omega_{1} \bar{N}_{1} / L_{1}}\right)^{\alpha+\beta}\left(\frac{\bar{N}_{2} / L_{2}}{\bar{N}_{1} / L_{1}}\right)^{1-\alpha-(1+\rho)(\beta+\gamma)}=\left(\frac{\left(1-\tau_{2}\right) a_{2} \xi\left(g_{2}\right)}{\left(1-\tau_{1}\right) a_{1} \xi\left(g_{1}\right)}\right)^{\beta+\gamma}
\end{array}\right.
$$

Proposition 3. When congestion cost is $\Omega_{j}>0$, a steady state with a gross population growth rate exceeding 1 does not exist. If a steady state with a population growth rate below 1 should exist, then that population growth rate is obtainable from equation (22) (the same also applies to the case in which $\Omega_{j}=0$ and $\bar{n} \geq 1$ ). Furthermore, if a steady state with a population growth rate of 1 does exist, then the population of each region in 
a steady state $\bar{N}_{j}(j=1,2)$ is obtainable from equation (23).

\section{Decentralized strategy in each region}

Here, equation (3) denotes the following: "in the presence of congestion cost associated with an increase in population density, the fertility of the region decreases with an increase in the population density. However, if the time cost is brought down by raising the level of childcare services provided in the region, the effect of the increased population density on fertility can be restrained." Therefore, we consider a decentralized strategy in which each $j$ region imposes payroll tax $\tau_{j}$ on its residents and uses its tax revenue as the financial resource to provide childcare services $g_{j}$ to its region. First, obtain the following from equation (8).

$$
\tau_{j}=g_{j}
$$

Then, consider a case of adopting a decentralized strategy in which each $j$ region provides childcare service $g_{j}$ entirely limited to its own region. If each region is assumed to choose a strategy that would maximize the utility of a representative agent in its region after migration, by considering the strategy of another region as given, from equations (14), (15), and (24), then each region would maximize the following, by considering strategy of another region as given.

$$
\begin{gathered}
\max _{g_{j}} \frac{\left(\left(1-g_{j}\right) a_{j} \xi\left(g_{j}\right)\right)^{\beta+\gamma}}{\left(\xi\left(g_{j}\right)+\frac{\alpha-\gamma}{1-\gamma} \Omega_{j} N_{j}(t) / L_{j}\right)^{\alpha+\beta}\left(N_{j}(t) / L_{j}\right)^{1-\alpha-(1+\rho)(\beta+\gamma)}} \\
\text { s.t. }\left\{\begin{array}{l}
\left(\frac{\xi\left(g_{2}\right)+\frac{\alpha-\gamma}{1-\gamma} \Omega_{2} N_{2}(t) / L_{2}}{\xi\left(g_{1}\right)+\frac{\alpha-\gamma}{1-\gamma} \Omega_{1} N_{1}(t) / L_{1}}\right)^{\alpha+\beta}\left(\frac{N_{2}(t) / L_{2}}{N_{1}(t) / L_{1}}\right)^{1-\alpha-(1+\rho)(\beta+\gamma)}=\left(\frac{\left(1-g_{2}\right) a_{2} \xi\left(g_{2}\right)}{\left(1-g_{1}\right) a_{1} \xi\left(g_{1}\right)}\right)^{\beta+\gamma} \\
\text { and } N_{1}(t)+N_{2}(t)=N^{\text {total }}(t)
\end{array}\right.
\end{gathered}
$$

Case 1. (when the child-rearing cost is dominant)

Equation (25) can be simplified as shown below because $\xi\left(g_{j}\right)>>\Omega_{j} N_{j}(t) / L_{j}$. 


$$
\begin{aligned}
& \bar{U}_{1}(t) \approx \frac{\left(\left(1-\tau_{1}\right) e_{1}(t)\right)^{\beta+\gamma}}{\xi\left(g_{1}\right)^{\alpha-\gamma}\left(N_{1}(t) / L_{1}\right)^{1-\alpha-\beta-\gamma}} \propto \frac{\left[\left(1-g_{1}\right) a_{1}\right]^{\beta+\gamma} L_{1}^{1-\alpha-(1+\rho)(\beta+\gamma)}}{\xi\left(g_{1}\right)^{\alpha-\gamma}\left[\frac{1}{1+\theta\left(t, g_{1}, g_{2}\right)}\right]^{1-\alpha-(1+\rho)(\beta+\gamma)}} \\
& \propto\left[H_{1} \varphi\left(g_{1}\right)^{\frac{1}{1-\alpha-(1+\rho)(\beta+\gamma)}}+H_{2} \varphi\left(g_{2}\right)^{\frac{1}{1-\alpha-(1+\rho)(\beta+\gamma)}}\right]^{1-\alpha-(1+\rho)(\beta+\gamma)} \\
& \bar{U}_{2}(t) \approx \frac{\left(\left(1-\tau_{2}\right) e_{2}(t)\right)^{\beta+\gamma}}{\xi\left(g_{2}\right)^{\alpha-\gamma}\left(N_{2}(t) / L_{2}\right)^{1-\alpha-\beta-\gamma}} \propto \frac{\left[\left(1-g_{2}\right) a_{2}\right]^{\beta+\gamma} L_{2}^{1-\alpha-(1+\rho)(\beta+\gamma)}}{\xi\left(g_{2}\right)^{\alpha-\gamma}\left[\frac{\theta\left(t, g_{1}, g_{2}\right)}{1+\theta\left(t, g_{1}, g_{2}\right)}\right]^{1-\alpha-(1+\rho)(\beta+\gamma)}} \\
& \propto\left[H_{1} \varphi\left(g_{1}\right)^{\frac{1}{1-\alpha-(1+\rho)(\beta+\gamma)}}+H_{2} \varphi\left(g_{2}\right)^{\frac{1}{1-\alpha-(1+\rho)(\beta+\gamma)}}\right]^{1-\alpha-(1+\rho)(\beta+\gamma)}
\end{aligned}
$$

Furthermore, when $\varphi\left(g_{j}\right) \equiv \frac{\left[1-g_{j}\right]^{\beta+\gamma}}{\xi\left(g_{j}\right)^{\alpha-\gamma}}, \quad H_{j}=a_{j}^{\frac{\beta+\alpha}{1-\alpha(1+\rho)(\beta+\gamma)}} L_{j}$, and $\xi\left(g_{j}\right) \equiv \xi_{0} \exp \left(-K g_{j}\right)$, then the level of childcare services that would optimize equations (25) and (26) is given as follows $(j=1,2)$ with a simple calculation as shown below.

where $K \geq \frac{\beta+\gamma}{(\alpha-\gamma)(1-\bar{\tau})}$ and $\Gamma_{1}(\rho)>0, \quad g_{j}=\bar{\tau}$

where $\frac{\beta+\gamma}{\alpha-\gamma}<K<\frac{\beta+\gamma}{(\alpha-\gamma)(1-\bar{\tau})}$ and $\Gamma_{1}(\rho)>0, g_{j}=1-\frac{\beta+\gamma}{(\alpha-\gamma) K}$

where $K \leq \frac{\beta+\gamma}{\alpha-\gamma}$ and $\Gamma_{1}(\rho)>0, g_{j}=0$

where $K<-\frac{(\beta+\gamma) \log (1-\bar{\tau})}{(\alpha-\gamma) \bar{\tau}}$ and $\Gamma_{1}(\rho)<0, \quad g_{j}=\bar{\tau}$

where $K \geq-\frac{(\beta+\gamma) \log (1-\bar{\tau})}{(\alpha-\gamma) \bar{\tau}}$ and $\Gamma_{1}(\rho)<0, \quad g_{j}=0$

Proposition 4. When the childcare cost is dominant $\left(\xi\left(g_{j}\right)>>\Omega_{j} N_{j}(t) / L_{j}\right)$, the level of childcare services that would maximize the utility of a representative agent in its own region is obtainable by equation (27), by considering the level of childcare services of another region as given. Because equation (27) is independent of the level of childcare services of another region, the level of childcare services obtained from equation (27) is a dominant strategy for each region.

Case 2. (when congestion cost is dominant) 
Equation (25) can be simplified as shown below because $\xi\left(g_{j}\right)<<\Omega_{j} N_{j}(t) / L_{j} \quad$ (same as case 1$)$ :

$$
\begin{aligned}
U_{j} & \propto \frac{\left\{\left(1-g_{j}\right) a_{j} \xi\left(g_{j}\right)\right\}^{\beta+\gamma}}{\Omega_{j}{ }^{\alpha+\beta}\left(N_{j}(t) / L_{j}\right)^{1+\beta-(1+\rho)(\beta+\gamma)}} \propto \frac{\left\{\left(1-g_{j}\right) a_{j} \xi\left(g_{j}\right)\right\}^{\beta+\gamma} L_{j}^{1+\beta-(1+\rho)(\beta+\gamma)}}{\Omega_{j}{ }^{\alpha+\beta}\left(\frac{1}{1+\theta}\right)^{1+\beta-(1+\rho)(\beta+\gamma)}} \\
& \left.\propto\left(\frac{L_{1}}{\Omega_{1} \frac{\alpha+\beta-(1+\rho)(\beta+\gamma)}{1+\beta}}\left(\left(1-g_{1}\right) a_{1} \xi\left(g_{1}\right)\right) \frac{\beta+\gamma}{1+\beta-(1+\rho)(\beta+\gamma)}+\frac{L_{2}}{\Omega_{2} \frac{\alpha+\beta}{1+\beta-(1+\rho)(\beta+\gamma)}}\left(1-g_{2}\right) a_{2} \xi\left(g_{2}\right)\right) \frac{\beta+\gamma}{1+\beta-(1+\rho)(\beta+\gamma)}\right)^{1+\beta-(1+\rho)(\beta+\gamma)}
\end{aligned}
$$

From equation (28), it is understood that to maximize the utility of its own region, each $j$ region simply needs to maximize $\left(\left(1-g_{j}\right) \xi\left(g_{j}\right)\right)^{\frac{\beta+\beta-(1+\rho)(\beta+\gamma)}{1+\gamma}}$. Therefore, if $\Gamma_{2}(\rho) \equiv 1+\beta-(1+\rho)(\beta+\gamma)>0, \quad g_{j}=0 \quad$ (no childcare services) $\left(\because \partial \xi\left(g_{j}\right) / \partial g_{j}<0\right)$. Additionally, it also means that, from equation (24), if $\Gamma_{2}(\rho)<0, g_{j}=\bar{\tau}$ (full childcare services) as the ceiling of payroll tax $\tau_{j}$ is $\bar{\tau}$ : as shown below $(j=1,2)$.

$$
\begin{cases}\text { where } \Gamma_{2}(\rho)>0, & g_{j}=0 \\ \text { where } \Gamma_{2}(\rho)<0, & g_{j}=\bar{\tau}\end{cases}
$$

Proposition 5. When the congestion cost is dominant $\left(\xi\left(g_{j}\right)<<\Omega_{j} N_{j}(t) / L_{j}\right)$, the level of childcare services that would maximize the utility of a representative agent in its own region is obtainable by equation (29), by considering the level of childcare services of another region as given. Because equation (29) is independent of the level of childcare services in another region, the level of childcare services obtained from equation (29) is a dominating strategy for each region.

Let us now seek a structural parameter $(\alpha, \beta, \gamma, \xi, \Omega)$ value that closely reflects the current Japanese economy, by assuming that region $j=1$ is the Tokyo metropolitan area (the metropolis and three surrounding prefectures, including Saitama, Chiba, and Kanagawa) and that region $j=2$ is the region other than the Tokyo metropolitan area, and $\xi\left(g_{j}\right)>>\Omega_{j} N_{j}(t) / L_{j}(j=1,2)$ as the time cost of child-rearing and congestion cost coefficient. First, the following is obtained from equations (3) and (4).

$$
\frac{n_{j}(t+1) z_{j}(t+1)}{\left(1-\tau_{j}\right) e_{j}(t)} \approx \frac{\gamma}{1-\gamma}
$$

Therein, $n_{j}(t+1)=1$. When the educational investment level per child $z_{j}(t+1)$ accounts for about $10 \%$ of the potential after-tax wage rate $\left(1-\tau_{j}\right) e_{j}(t) \quad\left(\right.$ e.g. when $\left(1-\tau_{j}\right) e_{j}(t)$ is 200 million yen, $z_{j}(t+1)$ is 20 million yen), roughly $\gamma=0.1$ is considered reasonable. From equation (5), the following is obtained. 


$$
\frac{c_{j}(t)}{\left(1-\tau_{j}\right) e_{j}(t)} \approx \frac{\beta}{1-\gamma}
$$

In that expression, if approximately half of the potential after-tax wage rate $\left(1-\tau_{j}\right) e_{j}(t)$ is allocated to consumption $c_{j}(t)$, excluding the child cost including opportunity cost of child-rearing $\left(z_{j}(t+1)+\left(1-\tau_{j}\right) e_{j}(t) \xi\left(g_{j}\right)\right)$ and residence cost $\left(R_{j}(t) q_{j}(t)\right)$, roughly $\beta=0.5$ is considered reasonable according to the equation above. From equation (6), the following is obtained.

$$
\frac{q_{j}(t) R_{j}(t)}{\left(1-\tau_{j}\right) e_{j}(t)} \approx \frac{(1-\alpha-\beta-\gamma)}{(1-\gamma)}
$$

Therein, if the residence cost $\left(R_{j}(t) q_{j}(t)\right)$ is approximately $20 \%$ of the potential after-tax wage rate $\left(1-\tau_{j}\right) e_{j}(t)$, then roughly $\alpha=0.2$ is considered reasonable.

Furthermore, the total fertility rate of Japan is currently at about 1.4, which means that $n_{j}(t+1)(j=1,2)$ is averaged at 0.7 . In this case, when the level of childcare services is $g_{j}=0$, it is assumed that the average lifetime wage loss ratio of husband and wife is $25 \%$ and $\xi\left(g_{j}\right)=\xi_{0} \exp \left(-K g_{j}\right)=0.25 \exp \left(-K g_{j}\right)$. In addition, if the population density in the Tokyo metropolitan area is assumed to be $1,200 / \mathrm{km}^{2}$ and population $N_{1}=3.6$ million, $L_{1}=4,000 \mathrm{~km}^{2}$, the total fertility rate is assumed to be $1.2\left(n_{1}=0.6\right)$, and the childcare service level is determined as the "potential capacity ratio of the childcare centers" (= capacity of childcare centers $\div$ female population aged between 25 and 44), which is at $8 \%$. Similarly, if the population density in areas other than the Tokyo metropolitan area is assumed to be $300 / \mathrm{km}^{2}$ and population $N_{2}=8.4$ million, $L_{2}=28,000 \mathrm{~km}^{2}$. Also, it is assumed that the total fertility rate is $1.5\left(n_{2}=0.75\right)$ and that the level of childcare services is $12 \%$. Furthermore, the maximum payroll tax rate (= maximum childcare services level) is assumed to be $\bar{\tau}=0.5$. Then, from equation (5), the following equations are valid.

$$
\begin{aligned}
& 0.6 \times 0.9 \times\left[0.25 \exp (-0.08 K)+\frac{0.1}{0.9} 1.2 \Omega\right]=0.1 \\
& 0.75 \times 0.9 \times\left[0.25 \exp (-0.12 K)+\frac{0.1}{0.9} 0.3 \Omega\right]=0.1
\end{aligned}
$$

When $\frac{0.1}{0.9} \Omega$ is eliminated from this simultaneous equation, the following is obtained.

$$
0.6 \times 0.9 \times[0.25 \exp (-0.08 K)+4(0.1 /(0.75 \times 0.9)-0.25 \exp (-0.12 K))]=0.1
$$

Then, $\Omega=0.074898$ and $K=4.502$ are obtained through numerical calculation of $K$.

That is, because $K<(\beta+\gamma) /(\alpha-\gamma)=6$, it can be understood from Proposition 4 that when $\rho<1 / 3, g_{j}=0(j=1,2)$. Furthermore, when $\rho>1 / 3$, the optimal childcare service level is $g_{j}=42.9 \%$ because $K<-(\beta+\gamma) \log (1-\bar{\tau}) /((\alpha-\gamma) \bar{\tau})$ is valid. When 
$\rho>1 / 3$ and if the "potential capacity ratio of the daycare centers" (= capacity of daycare centers $\div$ female population aged between 25 and 44) of the current Tokyo metropolitan area is $8 \%$. That of other areas is $12 \%$. These results suggest that the potential capacity ratio of the daycare centers in the two areas must be increased respectively by 5.3 times and by 3.5 times.

\section{Summary and future issues}

As described in this paper, we constructed a simple theoretical two-region OLG model by incorporating migration and land prices. Then we used the model to analyze the effects of population density and childcare services on fertility. Results elucidated the following three points.

First, in the presence of congestion cost associated with an increase in population density, the fertility rate of the region decreases concomitantly with increased population density. However, if the time cost is brought down by raising the level of the childcare services provided in the region, then the effect of the increased population density on fertility can be restrained.

Second, when the population size effect on productivity is slight, an improvement in childcare service availability and quality contributes to a heightened relative ratio of the population density. When the effect of population size on productivity is greater than a certain level, the relative ratio of the population density decreases if the relative ratio of the time cost of child-rearing decreases as a result of childcare service reform.

Third, where each region imposes payroll tax on its residents and uses its tax revenue as the financial resources to undertake a decentralized strategy of childcare service provision in its region, the level of childcare services that maximizes the utility of a representative agent in each region is independent of the childcare services of any other region. Therefore, changing the level of childcare services becomes a dominant strategy.

The following persist as issues for future studies:

The first issue is to perform empirical investigations of the effect of population size on productivity. If the model presented in this paper is reasonable, as Proposition 4 clarifies, then the magnitude of the effect of population size on productivity is an important consideration for determining the level of childcare service that maximizes the utility of a representative agent in each region. Therefore, performing empirical studies of the effects of population size on productivity is an important issue.

Second, the model presented in this paper encompasses only two regions and does not incorporate capital accumulation. Therefore, analyses to determine what 
modifications are required in Propositions 1-5 for cases in which area expansion and capital accumulation are regarded as important issues.

Third, for support policies for families with small children, not only improvement in childcare services but also a child allowance and expansion of educational assistance policies can be considered. However, the impact of such measures is not analyzed under the model used for this study. To clarify whether it is possible or not to reduce regional fertility disparities by increasing fertility through sufficient implementation of childcare support policies, which would enable the striking of a balance between child-rearing and work even in highly populated regions, comprehensive childcare support measures must be examined. Analyzing such measures remains as an important objective. 


\section{References}

Apps P. and Rees R. (2004) "Fertility, Taxation and Family Policy," Scandinavian Journal of Economics, vol. 106-4, pp. 745-763.

de Beers J., Deerenberg I. (2007) “An Explanatory Model for Projecting Regional Fertility Differences in the Netherlands," Population Research and Policy Review, vol. 26, pp. 511-528.

Galor O. and Weil D. (1996) "The Gender Gap, Fertility, and Growth," American Economic Review, vol. 86-3, pp. 374-387.

van Groezen, Leers and Meijdam (2003) "Social Security and Endogenous Fertility:

Pensions and Child Allowances as Siamese Twins," Journal of Public Economics vol. 87 , pp. 233-251.

Kulu H. (2013) "Why Do Fertility Levels Vary between Urban and Rural Areas?" Regional Studies, vol. 47(6), pp. 895-912.

O’Connell M. (1981) “Regional Fertility Patterns in the United States: Convergence or Divergence,” International Regional Science Review, vol. 6(1), pp. 1-14.

Sato Y. (2007) "Economic Geography, Fertility and Migration," Journal of Urban Economics, vol. 61, pp. 372-387.

Sato Y., Yamamoto K. (2005) "Population Concentration, Urbanization, and Demographic Transition," Journal of Urban Economics, vol. 58, pp. 45-61.

Sleebos J.E. (2003) "Low Fertility Rates in OECD Countries: Facts and Policy Responses," OECD Social, Employment and Migration Working Papers no. 15.

Tumen S. (2012) "Fertility Decisions and Endogenous Residential Sorting," Regional Science and Urban Economics, vol. 42, pp. 78-87.

Zhang J. (1997) "Fertility, Growth and Public Investments in Children," Canadian Journal of Economics, vol. 30-4, pp. 835-843.

Zhang J. (2002) "Urbanization, Population Transition, and Growth," Oxford Economic Papers, vol. 54, pp. 91-117. 